\title{
Clinical and histological predictive risk factors of atrial fibrillation in patients undergoing open-heart surgery
}

\author{
GRIGORE TINICA $^{1,2}$, VERONICA MOCANU ${ }^{3}$, FLORIN ZUGUN-ELOAE ${ }^{3}$ and DOINA BUTCOVAN ${ }^{1,3}$ \\ ${ }^{1}$ Department of Cardiovascular Surgery, 'Prof. Dr. George I.M. Georgescu' Institute of Cardiovascular Diseases, \\ Iasi 700503; Departments of ${ }^{2}$ Cardiac Surgery, and ${ }^{3}$ Morpho-Functional Sciences, 'Grigore T. Popa' \\ University of Medicine and Pharmacy, Iasi 700115, Romania
}

Received November 3, 2014; Accepted August 12, 2015

DOI: $10.3892 / \mathrm{etm} .2015 .2790$

\begin{abstract}
Postoperative atrial fibrillation (POAF) is a common complication subsequent to cardiac surgery. Various risk factors have been reported for the development of this complication; however, their precise role in POAF is unknown. In the present study, we attempted to identify clinical factors and histopathological changes in atrial tissue that may predict the development of POAF. Atrial tissue was sampled from 103 patients in sinus rhythm that had undergone open-heart surgery, including elective coronary artery bypass grafting (79.61\%) and heart valve surgery $(20.38 \%)$. Atrial surgical biopsies were obtained from the right atrial appendage at the site of cannulation, prior to cardiopulmonary bypass. Tissues were processed routinely for light microscopy, then stained with hematoxylin and eosin and sirius red. Microscopical exams were used to observe the atrial lesions and morphometry was conducted for quantification. In total, 37 patients (35.92\%) developed POAF and atrial lesions were identified in the majority of patients in the normal postoperative sinus rhythm and POAF groups, but were most common in the POAF patients. The most common risk factors involved in developing POAF were found to be: Age of $>60$ years, male gender, ejection fraction of $<50 \%$ and increased pulmonary hypertension. Furthermore, interstitial fibrosis and myocytolysis were the most common injuries identified. Abnormalities in atrial surgical biopsies may indicate the susceptibility of a patient to developing POAF. The present results suggest that the pre-existent alterations in the structure of the right atrium may be a major determinant in the development of POAF.
\end{abstract}

Correspondence to: Professor Veronica Mocanu, Department of Morpho-Functional Sciences, 'Grigore T. Popa' University of Medicine and Pharmacy, 16 Universitatii Street, Iasi 700115, Romania

E-mail: vmocanu@iasi.mednet.ro

Key words: atrial fibrillation, cardiac surgery, cardiopulmonary bypass, myocytolysis, interstitial fibrosis

\section{Introduction}

Atrial fibrillation (AF) is a common complication following cardiac operations that typically occurs within the first week following open-heart surgery, such as coronary artery bypass grafting (CABG) or heart valve surgery (1-3). Postoperative AF (POAF) is frequently considered to be a temporary problem; however, this complication results in significant adverse effects. POAF increases the risk of a cerebrovascular accident, extending the duration of hospital stay and the requirement for intensive care (4-6).

According to various studies, the risk factors associated with the development of POAF include right atrial manipulation, prolonged preoperative atrial conduction duration, atrial myocardial ischemia, prolonged aortic cross-clamping time and advanced age (7-9). Although nearly all patients present at least one of the aforementioned risk factors, $\sim 60 \%$ of patients undergoing cardiac surgery do not develop AF; thus, only certain patients seem to possess an inherent or acquired preoperative risk $(6,10)$. Therefore, these factors suggest that the major determinants of the risk for the development of POAF include the patient's preoperative status and condition of the atria, as well as the ischemia and stress induced by the surgical procedure.

The aim of the present study was to identify the markers of increased vulnerability in developing AF subsequent to CABG or heart valve surgery. The aim of the present study was to investigate the association among clinical (systemic hypertension), echocardiographic features (ejection fraction, left atrial dilation and pulmonary hypertension) and histological atrial lesions (cell atrophy, cell hypertrophy, interstitial fibrosis, myocytolysis, pericardial adiposity and inflammation) and the occurrence of POAF.

\section{Materials and methods}

Ethical approval. Ethical approval for the experiments conducted in the present study was obtained from the Institutional Board of 'Prof. Dr. George I.M. Georgescu', Institute of Cardiovascular Diseases (Iasi, Romania). All patients provided written informed consent.

Patient population. Between January 2010 and December 2011 a total of 103 selected patients underwent cardiac surgical proce- 
Table I. Modified protocol for routine histopathological examination of right atrial appendage specimens.

\section{A, Endocardium}

\begin{tabular}{lc}
\hline Features investigated & Possible observations \\
\hline Endocardial fibrosis & Absent/Present \\
Mononuclear exudates & Absent/Present \\
\hline
\end{tabular}

B, Myocardium/myocytes

\begin{tabular}{lc}
\hline Features investigated & Possible observations \\
\hline Myolytic vacuolation & Absent/Present \\
Size of vacuole & Mild/Severe \\
Frequency & $25 \%$ or $>25 \%$ \\
Cell hypertrophy & Absent/Present \\
Cell atrophy & Absent/Present \\
Lipofuscin & Absent/Present \\
Abnormal nuclei & Apoptotic figure \\
\hline
\end{tabular}

C, Interstitial myocardium

\begin{tabular}{lc}
\hline Features investigated & Possible observations \\
\hline Interstitial fibrosis & Absent/Present \\
Amount & Mild/Severe \\
Frequency & $25 \%$ or $>25 \%$ \\
Perivascular fibrosis & Absent/Present \\
\hline
\end{tabular}

D, Pericardium

Features investigated

Possible observations

Mononuclear exudate
Pericardial adiposity
Pericardial fibrosis

Absent/Present

Absent/Present

Absent/Present

Criteria modified from Ad et al (9).

dures requiring cardiopulmonary bypass $(\mathrm{CPB})$ at the 'Prof. Dr. George I.M. Georgescu' Institute of Cardiovascular Diseases. The patient population included 76 men and 27 women, with an age range of 42-77 years and a mean age of 61.8 years.

Cardiac assessment. All patients had undergone preoperative electrocardiography (SE 12; Edan Instruments, Inc., Nanshan, China) for selecting known cardiac patients in sinus rhythm and transthoracic echocardiography (Vivid E9 Electrocardiograph; GE Healthcare Bio-Sciences, Pittsburgh, PA, USA) was used to measure left atrial size along the anteroposterior diameter, left ventricular function and right atrial pressure

Sample collection. Right atrial appendage (RAA) tissue samples were collected from 103 patients in normal sinus rhythm, who were subjected to CABG (82 patients; $79.61 \%$ ) or heart valve surgery (21 patients; $20.38 \%$ ), prior to CPB. Right atrial appendage specimens were collected after opening of the pericardium, prior to cannulation of the right atrium. The age of the patients ranged between 42 and 77 years (mean age, 61.8 years), and 76 patients were females $(73.8 \%)$.

Histological and morphological examinations. Formalin-fixed atrial tissue was processed for paraffin embedding and the samples were cut into 4-6 $\mu \mathrm{m}$ sections. Next, histological sections were stained with hematoxylin and eosin and collagen-specific sirius red (Bio Optica Milano SpA, Milan, Italy). To highlight the cardiomyocyte (CM) nuclei, the sections stained with sirius red were re-stained with hematoxylin.

The specimens were examined histologically by a trained pathologist who was blinded to the patient characteristics and AF occurrence. Since atrial tissue analysis is not a routine procedure for pathologists, a previously-developed standard protocol was used for the examination of the RAA, as described by Ad et al (9) (Table I). The standard protocol was modified by adding the endocardial analysis step.

An Olympus BX40 light microscope (Olympus Corporation, Tokyo, Japan) was used for morphological evaluation. Histologically, the CM examination included analysis of the degree of myocytolysis, the existence of atrophy and other degenerative cell lesions. In the atrial interstitium, the degree of fibrosis was assessed. Furthermore, in the pericardium, the presence of mononuclear or fibrinous exudates, adiposity and fibrosis was investigated. Fibrosis was also evaluated at the endocardium. Semi-quantitative scales were used to evaluate the pathology of connective tissue components and atrial myocytes, as previously described by Ad et al $(8,9)$. Various degrees of lesions were identified and compared between the POAF and POSR groups.

Morphometric assessment. Atrial myocytes included the degree of vacuolization from loss of myofibrils. Vacuolization was scored as 0 or 1 ( 0 , absent; 1 , observed at any rate). The existence of hypertrophy and atrophy were rated between 0 and 1 as percentage of the number of hypertrophic or atrophic cells reported to total nucleated cell number, by assessing 10 high power field (HPF) sections from each group. Myocyte nuclear derangement encountered an evaluation of apoptotic pyknotic figures rated between 0 and 1 as percentage of the number of myolytic cells reported to total nucleated cell number, by assessing $10 \mathrm{HPF}$ sections from each group. The analysis of connective tissue components focused on fibrosis, rated between 0 and 1 as percentage of the fibrous interstitial area observed in the studied histological section area by assessing $10 \mathrm{HPF}$ sections per group, as described by Ad et al (9).

POAF and risk factors. POAF was identified on electrocardiogram in $37 / 103$ patients (35.9\%), and it occurred between 12 and $144 \mathrm{~h}$ after surgery (mean value, $45.1 \mathrm{~h}$ ). Following drug therapy, all patients affected by AF subsequently regained sinus rhythm prior to patient hospital discharge.

Monitoring. For post-operative AF detection, all patients were monitored daily until hospital discharge with standard 12-lead electrocardiography. Only AF episodes of $>15$ min 
Table II. Risk factors associated with normal POSR and POAF.

\begin{tabular}{lccc}
\hline Variable & POSR $(\%)$ & POAF $(\%)$ & P-value \\
\hline Age of $>60$ years & 42.5 & 75.6 & $\mathrm{P}<0.001$ \\
Male gender & 13.63 & 51.35 & $\mathrm{P}<0.001$ \\
Ejection fraction of $<50 \%$ & 1.51 & 75.67 & $\mathrm{P}<0.001$ \\
Left atrial dilatation of $>44 \mathrm{~mm}$ & 50 & 56.75 & $\mathrm{NS}$ \\
Pulmonary hypertension & 1.51 & 18.91 & $\mathrm{P}<0.001$ \\
Systemic hypertension & 77.27 & 72.97 & $\mathrm{NS}$ \\
Fibrinogen & 25.75 & 64.86 & $\mathrm{NS}$ \\
Hospital stay of $>14$ days & 39.39 & 29.72 & $\mathrm{NS}$ \\
Cell hypertrophy & 31.81 & 98.9 & $\mathrm{P}<0.001$ \\
Cell atrophy & 56.06 & 40.54 & $\mathrm{NS}$ \\
Interstitial fibrosis & 31.81 & 97.75 & $\mathrm{P}<0.001$ \\
Myocytolysis & 38.84 & 91.89 & $\mathrm{P}<0.001$ \\
Abnormal nuclei & 30.30 & 91.89 & $\mathrm{P}<0.01$ \\
Pericardial adiposity & 27.27 & 75.67 & $\mathrm{P}<0.001$ \\
Pericardial inflammatory infiltrate & 27.27 & 33.33 & $\mathrm{NS}$ \\
Endocardial fibrosis & 15.2 & 20.3 & $\mathrm{NS}$ \\
\hline
\end{tabular}

POSR, postoperative sinus rhythm; POAF, postoperative atrial fibrillation; NS, non-statistically significant difference $(P>0.05)$.

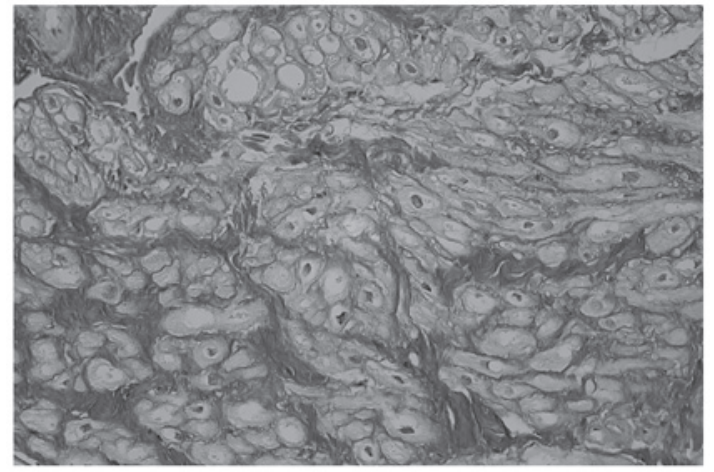

Figure 1. Representative specimen showing myocytolysis and fibrosis in a patient with postoperative atrial fibrillation. Postoperative atrial fibrillation samples exhibited cardiomyocytes (CMs) of various dimensions, with vacuoles of various sizes affecting the majority of the cells. Diffuse and large collagenous septa separating groups or isolated CMs with architecture disturbance were observed (picro-sirius red staining; magnification, $\mathrm{x} 40$ ).

duration were considered. Patients were diagnosed with POAF if interventional therapy (drug administration or electrical cardioversion) was required in order to restore the sinus rhythm. The majority of patients with POAF responded well to drug therapy, which included disopyramide, amiodarone and sotalol. In rare cases, including elderly or heart failure patients, electrical cardioversion was required to achieve sinus rhythm. Hospitalization durations of $>14$ days were required for all patients (mean, 19.93 days; range, 14-31 days).

Statistical analysis. The data were analyzed using the Excel software (Microsoft Corporation, Redmond, WA, USA). Pearson's $\chi^{2}$ test was used for categorical variables. A P-value of $<0.05$ was considered to indicate a statistically significant difference.

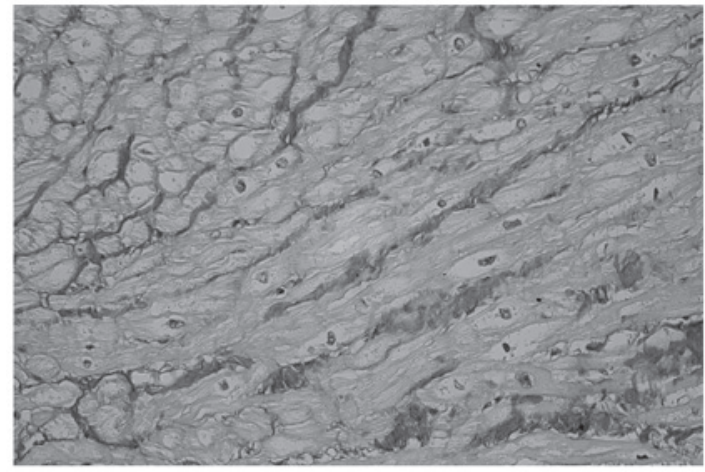

Figure 2. Representative specimen showing myocytolysis and fibrosis in a patient with postoperative sinus rhythm. Postoperative sinus rhythm samples exhibited vacuoles of various size in $<50 \%$ of the cells; a mild increase in cell size without significant atrial altered architecture. Increasing fibrotic bands separating large groups of cardiomyocyte exhibited focal distribution (picro-sirius red staining; magnification, x40).

\section{Results}

POAF and risk factors. POAF was identified using an electrocardiogram in $37 / 103$ patients $(35.92 \%)$, and it occurred between 12 and $144 \mathrm{~h}$ after surgery (mean, $45.1 \mathrm{~h}$ ). Following drug therapy, all patients affected by AF subsequently regained sinus rhythm prior to patient hospital discharge. The mean age of the patients with POAF (61.7 years) was higher compared with that of the patients remaining in sinus rhythm (SR) subsequent to cardiac surgery (58.7 years). In addition, only $42.5 \%$ of the patients with a postoperative sinus rhythm (POSR) were $>60$-years-old compared with $75.6 \%$ of patients with POAF. Thus, the results indicate that the risk of POAF is higher in patients with an age of $>60$ years $(\mathrm{P}<0.001)$. 
Several risk factors associated with the occurrence of POAF in cardiac surgery were analyzed, as shown in Table II. In the univariate analysis, the only independent clinical predictors of POAF risk were as follows: Age, $>60$ years; gender, male; ejection fraction, $<50 \%$; increased pulmonary hypertension; interstitial fibrosis; myocytolysis; cell hypertrophy; and pericardial adiposity $(\mathrm{P}<0.001$ for all factors) (Table II).

Left atrial size, measured by echocardiography, exceeded the upper limit (range, $25-44 \mathrm{~mm}$ ) in $56.75 \%$ of patients with POAF and $50 \%$ of patients with POSR. However, no correlation was identified between POAF and atrial dimensions $(\mathrm{P}>0.05)$.

Histopathological examination results. Upon histopathological examination, mild to severe myocytolysis was detected in the majority of the specimens, including $34 / 37$ patients in the POAF group (91.8\%; Fig. 1) and 25/66 patients in the POSR group (38.8\%; $\mathrm{P}<0.0001 ;$ Fig. 2). In addition, $\mathrm{CM}$ hypertrophy was identified in 98.9 and $31.81 \%$ of patients in the POAF and POSR groups, respectively $(\mathrm{P}<0.0001)$. $\mathrm{CM}$ atrophy was detected in $40.5 \%$ of patients in the POAF group and $56.0 \%$ in the POSR group. In the POAF group, the percentage of abnormal nuclei in each specimen was higher compared with that in the POSR group ( 91.8 vs. $30.3 \%$, respectively; $\mathrm{P}<0.01$ ). CMs with contraction band necrosis were rare findings in both groups, and were associated with ongoing IF only in the POAF patients.

Several histopathological abnormalities were encountered in the atrial interstitium of the two groups, but only IF exhibited a statistically significant difference. IF was detected in $97.7 \%$ of patients in the POAF group and $31.8 \%$ of patients in the POSR group $(\mathrm{P}<0.0001$; Figs. 1 and 2, respectively). Furthermore, no interstitial inflammatory infiltrate was observed, but a statistically significant difference in pericardial adiposity was detected between the two groups (76.7\% in POAF patients and $27.2 \%$ in POSR patients; $\mathrm{P}<0.0001$ ). A limited number of pericardial inflammatory foci, associated with localized pericardial fibrosis were observed in the POAF (33.3\%) and POSR (27.2\%) groups $(\mathrm{P}>0.05)$. The presence of pericardial inflammatory foci in both groups may indicate an ongoing healing process, associated with previous pericardial lesions. Endocardial fibrosis exhibited a focal and mild extension in the POAF (20.3\%) and POSR (15.2\%) groups ( $\mathrm{P}>0.05)$, which may be associated by the connective organization of small parietal thrombi.

\section{Discussion}

POAF is one of the most common causes of morbidity following cardiac surgery $(10,11)$. Although AF is a common postoperative complication, the incidence of POAF in patients undergoing cardiac surgery is unclear. Previously-reported incidence rates are between 10 and 65\%. In 1996, Mathew et al (10) reported an overall postoperative AFIB incidence of $27 \%$, while in 2001 Maisel et al (11) estimated that POAF occurs in 10-65\% of patients following cardiac surgery. This is a wide range since the definition of AF, detection methods, baseline patient characteristics and surgery type differ in previous studies (10). Maisel et al (11) estimated that POAF is $\sim 30 \%$ following standard CABG surgery and $40 \%$ subsequent to valve repairs or replacements, increasing to $\sim 50 \%$ after combined procedures.
Identifying the patients at a risk of developing POAF subsequent to cardiac surgery would result in the reduction of the incidence of POAF, as well as the prevention of undesired clinical consequences associated with this complication (4). Several studies have reported various risk factors for AF development following open-heart surgery $(3,4)$. Therefore, in addition to old age, numerous other risk factors have been identified by Almassi et al (3), such as chronic obstructive pulmonary disease, use of digoxin within 2 weeks prior to surgery, low resting pulse rate $(<80 \mathrm{bpm})$, high resting systolic blood pressure (>120 bpm) and use of inotropic agents for $>30$ min following the termination of CPB. In addition, Banach et al (12) identified further risk factors, including history of supraventricular arrhythmias, preoperative heart failure, operation with standard CABG technique and repeated revascularization. The common risk factors associated with POAF investigated in the present study, which were consisted with Aranki et al (5), were increasing age, male gender and hypertension.

The patient's age is the most common risk factor identified by previous studies. For instance, Hogue and Hyder (1) observed that in addition to age, valvular heart operation is the most consistently identified risk factor for cardiac arrhythmia. Similar to the present results (age, $\geq 60$ years; $\mathrm{P}<0.001$ ), Zaman et al (6) found that advanced age is markedly associated with postoperative AF (65.9 vs. 61.7 years; $\mathrm{P}<0.0005$ ). The frequency of this arrhythmia is increasing, possibly due to the rising proportions of elderly patients undergoing cardiac surgery. In the present study, the low incidence of POAF $(35.92 \%)$ may be associated with the relatively young mean age of the patients included in the present study (61.8 years).

Kitzman and Edwards (13) reported that fibrosis and atrophy in the atria, which are typical in older patients, as well as left atrial dilation, contribute to the susceptibility to develop POAF. However, the association between these features and AF occurring subsequent to cardiac surgery has not received considerable attention. Similar to other authors $(14,15)$, in the present study, we hypothesized that in the atrial myocardium of aged patients, atrophy and fibrosis may decrease the conductive tissue.

Data in the literature suggest that the left atrial size is an important factor in AF development (16-18). For instance, Henry et al (17) observed that AF is rare (3\%) when the left atrial size is $<44 \mathrm{~mm}$, but common $(54 \%)$ when the size is $>44 \mathrm{~mm}$. In addition, Ausma et al (18) noted that the AF risk increases by 1.4 times per 5 -mm increase in left atrial size. According to Li et al (19), atrial enlargement due to structural remodeling is a particularly important determinant of the occurrence of multiple-circuit reentry.

In the current study, histological lesions were observed in the majority of specimens, with generally higher values in POAF patients (hypertrophy, 98.9\%; interstitial fibrosis, 97.8\%; myocytolysis, 91.8\%; abnormal nuclei, 91.8\%; pericardial adiposity, 75,6\%) compared with those in POSR patients (hypertrophy, 31.8\%; interstitial fibrosis, 31.8\%; myocytolysis, $38.8 \%$; abnormal nuclei, $30.3 \%$; pericardial adiposity, $27.2 \%$ ). Consistent with the results of $\mathrm{Li}$ et al (19), the present results indicate that interstitial fibrosis is a significant risk factor for POAF development, having values $\sim 3$ times higher in POAF patients $(97.7 \%)$ compared with POSR patients (31.8\%). 
Usually, interstitial fibrosis is an expression of cardiac remodeling associated with various causes, such as chronic ischemia. In agreement with Boldt et al (20), we propose that fibrosis may explain the tissue anisotropy that results in inhomogeneous conduction, and may be responsible for the slow conduction and reentry that stabilizes AF.

A notable finding of the present study was the increased CM vacuolation observed in patients with POAF (91.8\%). Myocytolysis is a reversible, vacuolar degeneration of myocytes. Myocytolytic CMs are viable cells with a reduced function, due to loss of myofibrils. In addition, lesions may be associated with chronic ischemia. According to the results of Kitzman and Edwards (13) and Pirolo et al (21), CM vacuolation occurs as a consequence of the normal aging process or in response to the exposure to hypoxic stimuli in cardiac cells. Two previous studies by Ad et al $(8,9)$ identified myocytolysis as a key preoperative histopathological predictor for the development of POAF (65\%). In the present study, it was observed that the two patient groups (POAF, 91.8\%; POSR, $38.8 \%$ ) presented increased CM vacuolation as a possible arrhythmogenic substrate for the development of POAF; however, ultimately only certain patients developed POAF. Taking in consideration myolysis, Ausma et al (18) observed that the degree of myolysis and glycogen accumulation could increase with AF persistence. The enhanced accumulation of glycogen in the structurally altered atrial myocytes may imply an alteration of cellular metabolic status.

The majority of histologic changes, including atrophy and fibrosis, are characteristic of an ischemic myocardium. The presence of CM atrophy was not dominant in the current study, and CM hypertrophy was a compensatory lesion. Thus, atrophy and hypertrophy appear to induce background abnormality independently. Another two histological variables considered to be POAF predictors were pericardial adiposity and inflammation.

Although no interstitial atrial inflammation was identified in the current study, Ishii et al (22) noted that inflammation plays an important role in the pathogenesis of POAF, by altering atrial conduction, facilitating re-entry and predisposing to the development of POAF. The current results revealed only a small number of pericardial inflammatory foci, as possible AF trigger. Issac et al (23) considers that extracorporeal circulation contains enough systemic inflammatory mediators that may be, in part, responsible for the occurrence of POAF.

In the current analysis, a strong correlation was observed between extensive pericardial adiposity and POAF. Al Chekakie et al (24) and Batal et al (25), referring to the association between pericardial fat volume and AF, suggested that the local effects of proinflammatory cytokines released from the pericardial adipose tissue may be a potential mechanism for the development of AF.

In conclusion, in the present study, the preoperative status of atrial morphology was examined in correlation with various clinical risk factors. The results suggested that preoperative morphologic alterations, such as $\mathrm{CM}$ vacuolation and increased IF, may constitute a pathologic substrate and predictive factors for POAF development. However, the study had certain evident limitations. First, the number of patients in the study was small. Increasing the number of patients included in future studies would lead to a more accurate data analysis.
Secondly, a limitation of the study was participation of a single pathologist. In addition, only the right atrial appendage was sampled, while the left atrial tissue was not examined. Pulmonary veins and left atrial tissue are known to be critical regions in the initiation and maintenance of AF. However, as the left atrium is more difficult to access during surgery due to its posterior position, the investigation of this area may require the use of necropsy samples or an experimental animal study.

\section{References}

1. Hogue CW Jr and Hyder ML: Atrial fibrillation after cardiac operation: Risks, mechanisms and treatment. Ann Thorac Surg 69: 300-306, 2000.

2. Creswell LL, Schuessler RB, Rosenbloom M and Cox JL: Hazards of postoperative atrial arrhythmias. Ann Thorac Surg 56: 539-549, 1993.

3. Almassi GH, Schowalter T, Nicolosi AC, Aggarwal A, Moritz TE, Henderson WG, Tarazi R, Shroyer AL, Sethi GK, Grover FL and Hammermeister KE: Atrial fibrillation after cardiac surgery: A major morbid event? Ann Surg 226: 501-511, 1997.

4. Cox JL: A perspective of postoperative atrial fibrillation in cardiac operations. Ann Thorac Surg 56: 405-409, 1993.

5. Aranki SF, Shaw DP, Adams DH, Rizzo RJ, Couper GS, VanderVliet M, Collins JJ Jr, Cohn LH and Burstin HR: Predictors of atrial fibrillation after coronary artery surgery. Current trends and impact on hospital resources. Circulation 94: 390-397, 1996.

6. Zaman AG, Archbold RA, Helft G, Paul EA, Curzen NP and Mills PG: Atrial fibrillation after coronary artery bypass surgery: A model for preoperative risk stratification. Circulation 101: 1403-1408, 2000.

7. Groves PH and Hall RJ: Atrial tachyarrhythmias after cardiac surgery. Eur Heart J 12: 458-463, 1991.

8. Ad N, Snir E, Vidne BA and Golomb E: Potential preoperative markers for the risk of developing atrial fibrillation after cardiac surgery. Semin Thorac Cardiovasc Surg 11: 308-313, 1999.

9. Ad N, Snir E, Vidne BA and Golomb E: Histologic atrial myolysis is associated with atrial fibrillation after cardiac operation. Ann Thorac Surg 72: 688-693, 2001.

10. Mathew JP, Parks R, Savino JS, Friedman AS, Koch C, Mangano DT and Browner WS: Atrial fibrillation following coronary artery bypass graft surgery: Predictors, outcomes and resource utilization. Multi Center Study of Perioperative Ischemia Research Group. JAMA 276: 300-306, 1996.

11. Maisel WH, Rawn JD and Stevenson WG: Atrial fibrillation after cardiac surgery. Ann Intern Med 135: 1061-1073, 2001.

12. Banach M, Rysz J, Drozdz JA, Okonski P, Misztal M, Barylski M, Irzmanski R and Zaslonka J: Risk factors of atrial fibrillation following coronary artery bypass grafting: A preliminary report. Circ J 70: 438-441, 2006.

13. Kitzman DW and Edwards WD: Age-related changes in the anatomy of the normal human heart. J Gerontol 45: M33-M39, 1990.

14. Lie JT and Hammond PI: Pathology of the senescent heart: Anatomic observations on 237 autopsy studies of patients 90 to 105 years old. Mayo Clin Proc 63: 552-564, 1988.

15. Goette A, Juenemann G, Peters B, Klein HU, Roessner A, Huth C and Röcken $\mathrm{C}$ : Determinants and consequences of atrial fibrosis in patients undergoing open heart surgery. Cardiovasc Res 54: 390-396, 2002.

16. Vaziri SM, Larson MG, Benjamin EJ and Levy D: Echocardiographic predictors of nonrheumatic atrial fibrillation. The framingham heart study. Circulation 89: 724-730, 1994.

17. Henry WL, Morganroth J, Pearlman AS, Clark CE, Redwood DR, Itscoitz SB and Epstein SE: Relation between echocardiographically determined left atrial size and atrial fibrillation. Circulation 53: 273-279, 1976.

18. Ausma J, Litjens N, Lenders MH, Duimel H, Mast F, Wouters L, Ramaekers F, Allessie M and Borgers M: Time course of atrial fibrillation-induced cellular structural remodeling in atria of the goat. J Mol Cell Cardiol 33: 2083-2094, 2001.

19. Li D, Fareh S, Leung TK and Nattel S: Promotion of atrial fibrillation by heart failure in dogs: Atrial remodeling of a different sort. Circulation 100: 87-95, 1999. 
20. Boldt A, Wetzel U, Lauschke J, Weigl J, Gummert J, Hindricks G, Kottkamp $\mathrm{H}$ and Dhein S: Fibrosis in left atrial tissue of patients with atrial fibrillation with and without underlying mitral valve disease. Heart 90: 400-405, 2004.

21. Pirolo JS, Hutchins GM and Moore GW: Myocyte vacuolization in infarct border zones is reversible. Am J Pathol 121: 444-450, 1985.

22. Ishii Y, Schuessler RB, Gaynor SL, Yamada K, Fu AS, Boineau JP and Damiano RJ Jr: Inflammation of atrium after cardiac surgery is associated with inhomogeneity of atrial conduction and atrial fibrillation. Circulation 111: 2881-2888, 2005.
23. Issac TT, Dokainish H and Lakkis NM: Role of inflammation in initiation and perpetuation of atrial fibrillation: A systematic review of the published data. J Am Coll Cardiol 50: 2021-2028, 2007.

24. Al Chekakie MO, Welles CC, Metoyer R, Ibrahim A, Shapira AR, Cytron J, Santucci P, Wilber DJ and Akar JG: Pericardial fat is independently associated with human atrial fibrillation. J Am Coll Cardiol 56: 784-788, 2010.

25. Batal O, Schoenhagen P, Shao M, Ayyad AE, Van Wagoner DR Halliburton SS, Tchou PJ and Chung MK: Left atrial epicardial adiposity and atrial fibrillation. Circ Arrhythm Electrophysiol 3: 230-236, 2010. 